\title{
Multi component coupling reactions of $N$-acetyl-2-azetine
}

\author{
Paul J. Stevenson,* Mark Nieuwenhuyzen, and Daire Osborne \\ School of Chemistry and Chemical Engineering, The Queen's University of Belfast, Belfast, \\ Northern Ireland, BT9 $5 A G$ \\ E-mail:p.stevenson@qub.ac.uk
}

\begin{abstract}
$\mathrm{N}$-Acetyl-2-azetine undergoes Lewis acid catalysed formal [4+2]-cycloaddition with imines derived from aromatic amines to initially give an approximately 1:1 mixture of exo-endodiastereoisomeric 1-(2a,3,4,8b-tetrahydro-2H-1,4-diaza-cyclobuta[ $a]$ naphthalen-1-yl)-ethanone cycloadducts which were detected by proton NMR spectroscopy. These products, which were too unstable to isolate, and characterise, reacted further with aromatic amines to give 2,3,4trisubstituted tetrahydroquinolines in good to excellent yield, predominantly as a single diastereoisomer, with the minor diastereoisomer converting to the major diastereoisomer on silica. The cycloaddition was irreversible and a mechanism is presented for the formation of the major diastereoisomer from the mixture of diastereoisomeric intermediates. A range of conditions is described for converting the 2,3,4-trisubsitituted tetrahydroquinolines into 2,3disubstituted quinolines.
\end{abstract}

Keywords: Imino Diels-Alder reaction, Lewis acid catalysis, cycloaddition, multi-component coupling reactions MCR

\section{Introduction}

The imino-Diels-Alder reaction, originally described by Povarov, ${ }^{1}$ involves coupling of imines, derived from aromatic amines, or their surrogates, with electron rich alkenes and has emerged as a powerful tool for the synthesis of tetrahydroquinolines, and the subject has been reviewed several times. ${ }^{2,3}$ The original reaction was catalysed by boron trifluoride etherate ${ }^{4,5}$ but more recently it was demonstrated that a wide range of Lewis acids, ${ }^{6-9}$ or protic acids ${ }^{10-12}$ also catalyse this process. No catalyst is required if the reaction is carried out in an ionic liquid ${ }^{13}$ or 2,2,2trifluoroethanol. ${ }^{14}$ There does not appear to be one generic mechanism to adequately explain all reactions in this class, with some substrates appearing to react by a formal concerted [4+2] cycloaddition $^{15}$ whilst others appear to react via a stepwise process with discrete ionic intermediates. $^{16,17}$ 
Recently, during endeavors directed towards the synthesis of the alkaloid martinelline $\mathbf{1}{ }^{18}$ we $^{19-21}$ and others ${ }^{22-24}$ were investigating [4+2]-imino-Diels-Alder reactions of imines derived from aromatic amines with five membered ring cyclic enamides as a method for rapidly assembling the central heterocyclic core of this unusual alkaloid, Scheme 1. Although this chemistry proceeds well, it is known that tetrahydroquinolines with a hetero-substituent at the 4position are potentially labile, particularly in the presence of protic or Lewis acid, and are likely to lose the oxygen or nitrogen substituent ultimately leading to quinoline products. Indeed, the original reactions of imines derived from aromatic amines with enol ethers gave exclusively quinoline products by an elimination-oxidation sequence. ${ }^{4,5}$ In our case the stability of the hexahydropyrroloquinoline 2 was determined by the substituent at C-2, (tetrahydroquinoline numbering), and substrates with a carboethoxy group at this position were particularly unstable and prone to fragment to give the quinoline product 3a. ${ }^{21}$ When an NMR sample of 2a was stored overnight, and the spectrum recorded, compound 2a was quantitatively converted to the quinoline 3a. Similar tandem fragmentation oxidations have been noted when there is an ether at C-4 though harsher acidic conditions were required. ${ }^{25}$ More recently it has been demonstrated that ceric ammonium nitrate ${ }^{26}$ also affects an elimination-oxidation of 4-amido-substituted tetrahydroquinolines, though in this case the oxidation may precede the elimination. However, in our case, the reaction was limited to substrates containing a carboethoxy substituent at C-2 with compound $\mathbf{2 b}$ indefinitely stable in deuteriochloroform and conditions could not be found to effect elimination-oxidation to give $\mathbf{3 b}$.<smiles>CC(C)=CCNC(=N)NCCC[C@H]1Nc2ccccc2C2C1CCN2C(=N)NCC=C(C)C</smiles><smiles>[R]C=Nc1ccccc1</smiles>

Scheme 1. Elimination-oxidation approach to 2,3-disubstituted quinolines.

$N$-Acetyl-2-azetine 6, participates in Diels-Alder reactions and undergoes [2+2] cycloreversions followed by intramolecular Diels-Alder reactions. ${ }^{27-29}$ It was envisaged that if 
this compound participated in imino-Diels-Alder reactions, with an aromatic imine, then the products would be much more prone to participate in elimination-oxidation reactions regardless of the substituent at C-2. The initial results of imino-Diels-Alder reactions of $N$-acetyl-2-azetine, with imines derived from aromatic amines, have been previously reported in preliminary form $^{30,31}$ and we now present full details of this chemistry.

\section{Results and Discussion}

$N$-Acetyl-2-azetine 6 was prepared by the literature procedure ${ }^{28}$ as outlined in Scheme 2. Although this compound was previously known, no detailed experimental procedures for the final two steps were reported. Alcohol 4 was converted into $N$-acetyl-2-azetine 6 uneventfully in $32 \%$ overall yield by formation of the mesylate followed by elimination using potassium tertiary butoxide as base. The moderate overall yield can be attributed to the instability of the azetine and water solubility of azetine and mesylate intermediate 5. This compound proved to be remarkably stable for a cyclic enamide, and could be stored indefinitely at $-5^{\circ} \mathrm{C}$ in a refrigerator.

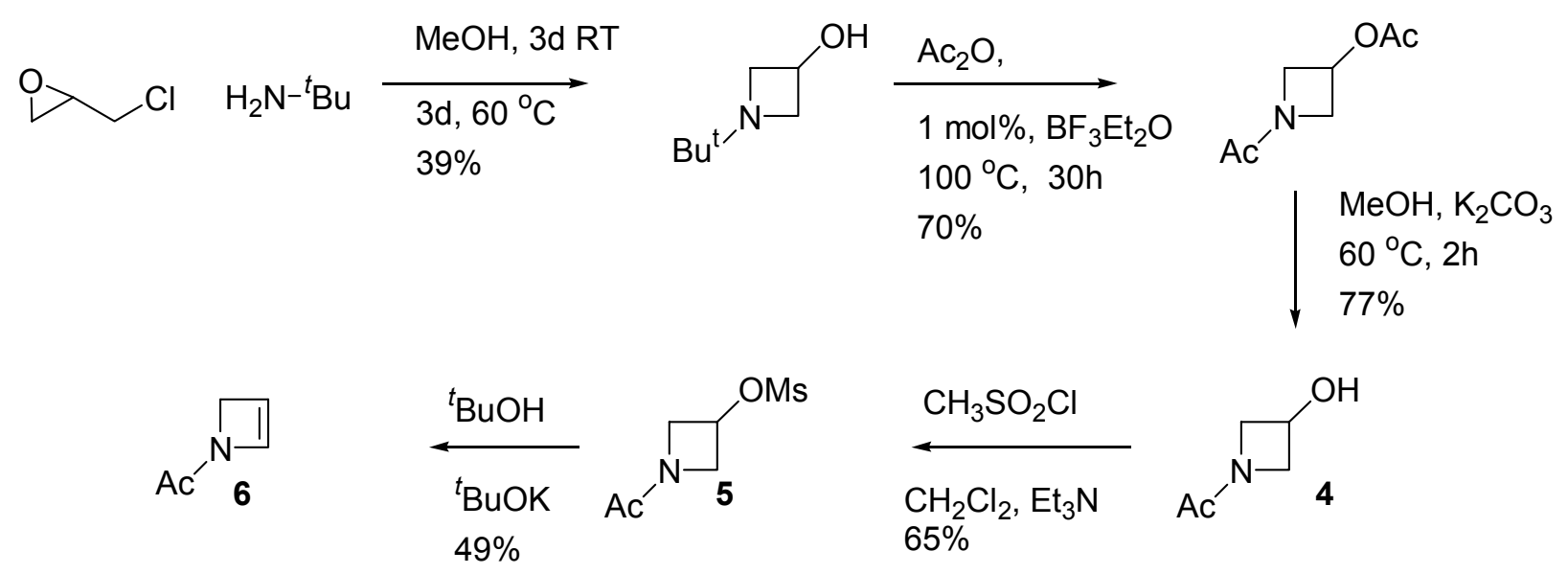

Scheme 2. Preparation of $N$-acetyl-2-azetine.

Initially the imino Diels-Alder reaction of $N$-acetyl-2-azetine 6 with imine 7a, using yttrium triflate as Lewis acid, proved extremely problematic. Although both starting materials were rapidly consumed, proton NMR analysis of the crude reaction mixture indicated that the reaction was not clean and that many products had formed from which quinoline 11a could be sometimes isolated. The reproducibility with respect to yield, was poor and ranged from $0-39 \%$. In order to identify the troublesome step, in this multi step coupling sequence, the reaction was carried out in d-3-acetonitrile with yttrium triflate as catalyst and directly monitored by proton NMR spectroscopy. Gratifyingly, the imino Diels-Alder reaction proceeded rapidly, cleanly, and completely regioselectively at room temperature. After one hour all the $N$-acetyl-2-azetidine 6 was consumed and a 55:45 mixture of exo-endo-diastereoisomeric 1-(2a,3,4,8b-tetrahydro- $2 \mathrm{H}$ - 
1,4-diaza-cyclobuta $[a]$ naphthalen-1-yl)-ethanones 8a were present, Scheme 3. These compounds were readily identified by the similarity of spectra to the more stable exo/endohexahydropyrolloquinolines $\mathbf{2 b}$, previously isolated and characterised, ${ }^{21}$ with ring junction proton $\mathrm{H} 8 \mathrm{~b}$ coming into resonance at the distinctive positions of $\delta 5.38$ and 5.20 as doublets for both isomers. On standing overnight, and recording the NMR spectrum, all peaks for tricyclic compound $\mathbf{8}$ had gone and new extremely broad peaks were evident indicating that the material had possibly polymerised. On standing for several days, or heating for four hours no quinoline product 11a was detected by proton NMR spectroscopy, strongly suggesting that the quinolines could be a product of workup. This raises the interesting question as to what the structure of the suspected polymeric material was. One interesting idea was that 1-(3-phenyl-2a,3,4,8btetrahydro-2H-1,4-diaza-cyclobuta $[a]$ naphthalen-1-yl)-ethanone $8 \mathbf{a}$ was undergoing a ring opening reaction, facilitated by the tetrahydroquinoline nitrogen, to iminium salt 9 which then reacted with additional 8a to give a dimer 10, which then oligomerised and ultimately polymerised by a repetition of the aforementioned events. To test this theory, an additional NMR experiment was run which contained aromatic amine. Again the exo/endo-cycloadducts 8a, were rapidly formed inside one hour at room temperature, with the primary aromatic amine having no detrimental effect on the catalyst, and these cycloadducts slowly reacted with the aromatic amine, typically over twelve hours, and gave the 2,3,4-tetrahydroquinolines diastereoisomers 12a and 13a as the sole reaction products. With conditions worked out on how to perform the multicomponent coupling reaction, a range of imines $7 \mathbf{a}-\mathbf{f}$ were reacted with $N$-acetyl-2-azetine and aromatic amine, from which the imine was derived, and the results are summarized in Table 1. In most cases the reaction proceeded smoothly and gave a crude product as a mixture of diastereoisomers 12 and 13. However, unless the silica gel used for chromatography was prewashed with triethylamine, only the major diastereoisomer 12 could be isolated. Clearly these diastereoisomers are interconverting. The low yield for entry $\mathbf{f}$ probably reflects the instability of the imine and the reaction failed completely for imines derived from aliphatic aldehydes with only intractable material produced.

Table 1. Tetrahydroquinolines by three component coupling reactions

\begin{tabular}{cccccc}
\hline Entry & $\mathrm{R}^{1}$ & $\mathrm{R}^{2}$ & $\mathrm{R}^{3}$ & $\mathbf{1 2 : 1 3}$ ratio & Yield 12\% \\
\hline $\mathrm{a}$ & $\mathrm{H}$ & $\mathrm{Ph}$ & $\mathrm{H}$ & $95: 5$ & 91 \\
$\mathrm{~b}$ & $\mathrm{OMe}$ & $\mathrm{Ph}$ & $\mathrm{OMe}$ & $90: 10$ & 85 \\
$\mathrm{c}$ & $\mathrm{Me}$ & $4-\mathrm{NO}_{2} \mathrm{Ph}$ & $\mathrm{Me}$ & $99: 1$ & $28^{*}$ \\
$\mathrm{~d}$ & $\mathrm{H}$ & $4-\mathrm{MeOPh}$ & $\mathrm{H}$ & $90: 10$ & 97 \\
$\mathrm{e}$ & $\mathrm{H}$ & $\mathrm{CO}_{2} \mathrm{Et}$ & $\mathrm{H}$ & $95: 5$ & 97 \\
$\mathrm{f}$ & $\mathrm{OMe}$ & $\mathrm{CH}=\mathrm{CHPr}^{2}$ & $\mathrm{OMe}$ & $92: 8$ & 30 \\
$\mathrm{~g}$ & $\mathrm{Me}$ & $4-\mathrm{NO}_{2} \mathrm{Ph}$ & $\mathrm{H}$ & $96: 4$ & 66 \\
\hline
\end{tabular}

* Another $30 \%$ of the mass balance was accounted for, by precipitation of $\mathbf{8 c}$. 


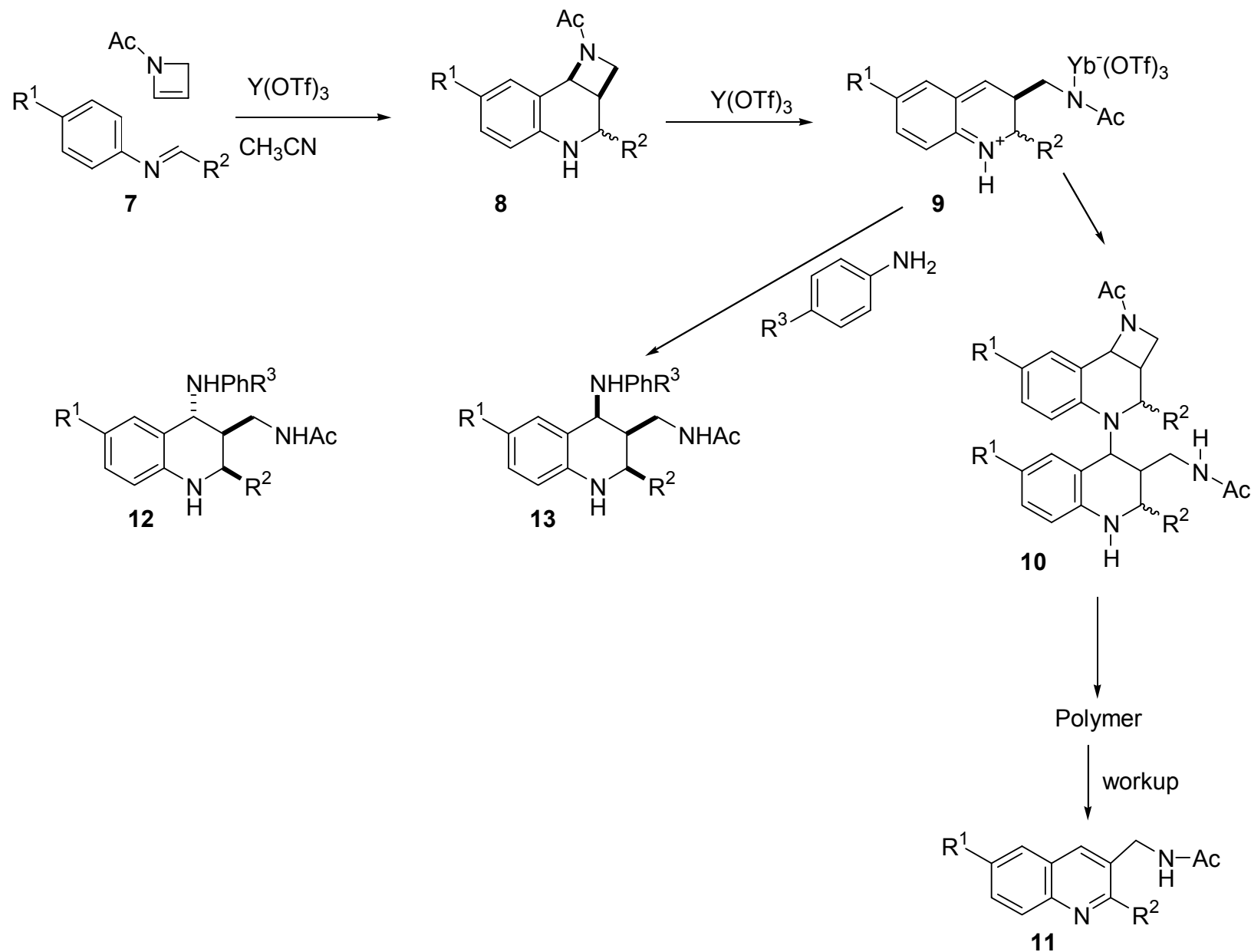

Scheme 3. Multicomponent coupling of amine, imine, and $N$-acetyl-2-azetine.

Assigning the relative stereochemistry of the major diastereoisomer $\mathbf{1 2}$ proved difficult, by proton NMR spectroscopy, as the preferred conformation of the saturated ring was also unknown. This problem was solved by single crystal X-ray crystallography on $\mathbf{1 2 d}^{32}$ and the other members of the series by comparison of their NMR spectra with that of 12d. Interestingly, this molecule adopts a conformation which puts the two substituents at C-3 and C-4 transdiaxial, presumably to minimise 1,3 -allylic strain of $\mathrm{H}-5$ with the aniline substituent at $\mathrm{C}-4,{ }^{33}$ with the phenyl substituent at $\mathrm{C}-2$ equatorial. The molecule adopts the same conformation in solution with a coupling constant $\mathrm{J}_{\mathrm{H} 3-\mathrm{H} 4}$ of less than the line width, $1.4 \mathrm{~Hz}$, which is consistent with both H-3 and H-4 being trans-diequatorial. Although the minor diastereoisomers 13a-f could be detected by proton NMR spectroscopy, in the crude reaction mixtures, their isolation and purification proved to be extremely challenging. Using standard flash chromatography these minor isomers were never isolated, probably due to an equilibration of $\mathbf{1 3}$ to $\mathbf{1 2}$ on the column. However, in one case (entry a), the column was pre-washed with triethylamine, prior to chromatography, and 13a was isolated in 3\% yield. It also proved difficult to determine the relative stereochemistry of this diastereoisomer by proton NMR spectroscopy. The coupling 
constants $\mathrm{J}_{\mathrm{H} 2-\mathrm{H} 3}$ and $\mathrm{J}_{\mathrm{H} 3-\mathrm{H} 4}, 2.5 \mathrm{~Hz}$ and less than $1 \mathrm{~Hz}$ respectively, were very similar to that of the major diastereoisomer, $2.8 \mathrm{~Hz}$ and less than $1.4 \mathrm{~Hz}$. However, saturation at $\mathrm{H}-2$ gave a $4.4 \%$ and $3.7 \%$ nOe to $\mathrm{H}-3$ and $\mathrm{H}-4$ respectively, supporting the proposed structure of 13a, and indicating that both $\mathrm{H}-2$ and $\mathrm{H}-4$ had a cis-1,3-diaxial relationship.

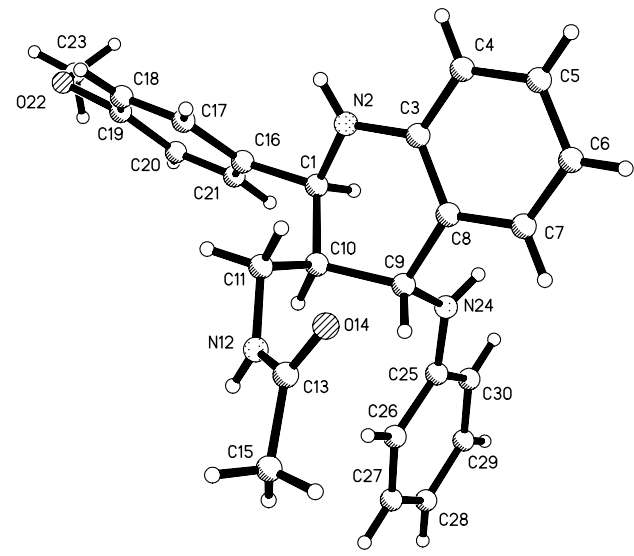

Figure 1. X-Ray structure of diastereoisomer 4d depicting relative stereochemistry and conformation.

In the case of imine $7 \mathbf{c}$ the isolated yield of product was low, 28\%. The reason for this was that the intermediate cycloadduct $\mathbf{8 c}$ was not very soluble in acetonitrile and precipitated during the course of the reaction. It proved possible to filter this solid and it was soluble enough in d-3acetonitrile for a proton NMR spectrum to be recorded. When $p$-methoxyaniline was added to the NMR sample and the reaction monitored by proton NMR spectroscopy, nothing happened, with cycloadduct $\mathbf{8 c}$ being stable under these reaction conditions. When one crystal of yttrium triflate was added to the NMR sample the reaction proceeded and gave a 99:1 mixture of diastereoisomers 12c:13c. These results prove that the ring opening reaction of $\mathbf{8 c}$ with aromatic amine was Lewis acid catalysed. When imine 7c was reacted with $\mathrm{N}$-acetyl-2-azetidine and aniline, a complex mixture of products resulted due to imine equilibration, giving rise to two different imines and amines leading to a permutation of four different products plus their minor diastereoisomers. However, when precipitated cycloadduct $\mathbf{8 c}$ was reacted with aniline under standard reaction conditions this gave $\mathbf{1 2} \mathrm{g}$ as the sole reaction product. These findings prove that the imino Diels-Alder reaction giving rise to $\mathbf{8 c}$ is irreversible. This then raises the interesting question as to how a single diastereoisomeric tetrahydroquinoline product, with respect to centres C-2 and C-3, is formed from a 55:45 mixture of exo-endo diastereoisomers 8. Chiral centres $\mathrm{C}-2$ or $\mathrm{C}-3$, or both, must be epimerising at some stage in the subsequent sequence and the most likely place for this to happen is at the stage of intermediate $\mathbf{9}$, prior to reaction with aromatic amine. 
With tetrahydroquinolines $\mathbf{1 2}$ at hand, attempts were made to achieve the initial goal and convert these to 2,3-disubstituted quinolines, in a reproducible manner. This transformation was readily achieved by stirring with acetic acid, heating with yttrium triflate, or oxidation with DDQ and the results are summarised in Scheme 4. Presumably, the reason for the success in this sequence is that monomeric tetrahydroquinolines $\mathbf{1 2 b}, \mathbf{c}, \mathbf{e}$ are more reactive than their polymeric counterparts that would have resulted if the aromatic amine was omitted from the initial cycloaddition.

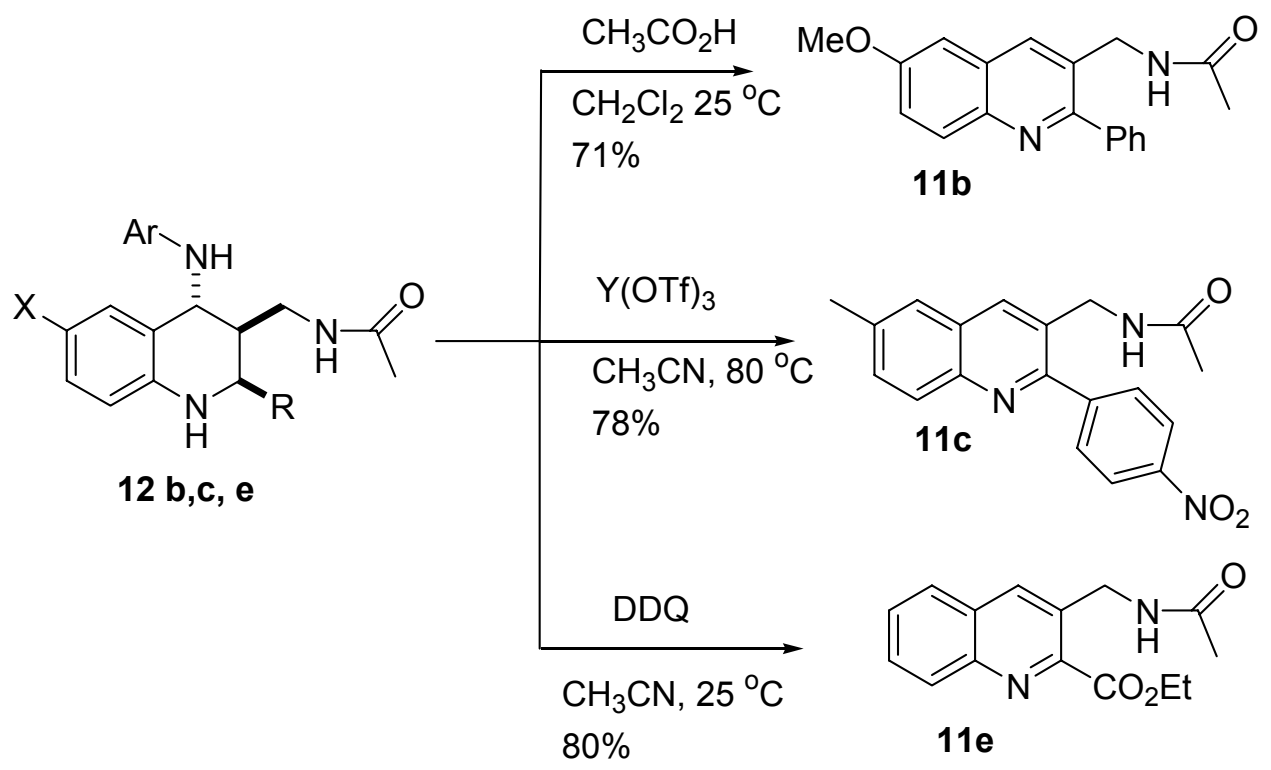

Scheme 4. Elimination, oxidation reactions of tetrahydroquinolines.

The second reaction, heating with yttrium triflate, was particularly attractive, because once the initial multicomponent coupling is complete, heating the mixture initiates the eliminationoxidation sequence turning this into a one pot procedure. The results of these one pot couplings to give 2,3-disubstituted quinolines are summarised in Scheme 5. Although it is known that imino Diels-Alder reactions of meta-substituted aromatic imines can be completely regioselective, ${ }^{34,35}$ in the the case of imine $\mathbf{7 h}$ the regioselectivity for the cycloaddition was poor. 


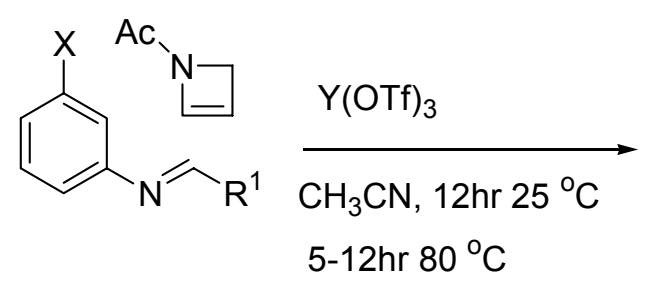

7a $X=H ; R^{1}=P h$

$7 \mathrm{~h} X=\mathrm{Br} ; \mathrm{R}^{1}=\mathrm{Ph}$

$7 \mathrm{j} X=\mathrm{H} ; \mathrm{R}^{1}=4-\mathrm{OHPh}$

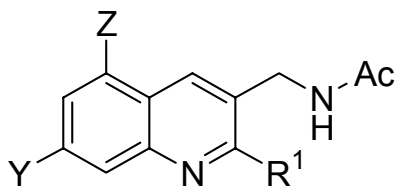

11a $Y=Z=H ; R^{1}=P h 85 \%$

11h $\mathrm{Y}=\mathrm{H} ; \mathrm{Z}=\mathrm{Br} ; \mathrm{R}^{1}=\mathrm{Ph} 47 \%, 11 \mathrm{i} \mathrm{Y}=\mathrm{Br} ; \mathrm{Z}=\mathrm{H} ; \mathrm{R}^{1}=\mathrm{Ph} 30 \%$;

11j $Y=H ; Z=H ; R^{1}=4-O H P h 62 \%$

Scheme 5. 2,3-Disubstituted quinolines via multicomponent coupling.

In conclusion, we have demonstrated that the multicomponent reaction comprising of an imino Diels-Alder reaction, fragmentation-oxidation to give 2,3-disusbtsituted quinolines, is facilitated by the addition of aromatic amines, which functions by preventing unstable intermediates from polymerising.

\section{Experimental Section}

General Procedures. Melting points were recorded using a Kofler hot stage apparatus and are uncorrected. I.R spectra were recorded on a Perkin-Elmer Model 983G instrument coupled to a Perkin-Elmer 3700 Data Station as potassium bromide (KBr) disks, or films (liquids). ${ }^{1} \mathrm{H}$ nuclear magnetic resonance (NMR) spectra were recorded at $300 \mathrm{MHz}$ using Bruker DPX 300 and at $500 \mathrm{MHz}$ using Bruker DRX 500 NMR spectrometers. Chemical shifts are given in parts per million $(\delta)$ down field from tetramethylsilane as internal standard and coupling constants are given in Hertz. Unless otherwise stated, deuteriochloroform was used as solvent. Spectra splitting patterns are designated as s: singlet, $\mathrm{d}$ : doublet, $\mathrm{t}$ : triplet, q: quartet, m: multiplet and br: broad. Mass spectra were recorded using Double Focusing Triple Sector VG Auto Spec and accurate molecular masses were determined by the peak matching method using perfluorokerosene as standard reference and were accurate to within +/-0.006 a.m.u. Analytical tlc was carried out on Merck Kielselgel $60_{254}$ plates and the spots visualised using a Hanovia Chromatolite uv lamp. Flash chromatography was effected using Merck Kielselgel 60 (230-400 mesh).

Imines $7 \mathbf{a}-\mathbf{f}^{36-39}$ were prepared by condensation of aldehyde $(2.6 \mathrm{mmol})$ with amine $(2.6 \mathrm{mmol})$ in benzene $(30 \mathrm{~mL})$ with azeotropic removal of water.

Methanesulfonic acid 1-acetyl-azetidin-3-yl ester 5. Methanesulphonyl chloride ( $0.17 \mathrm{~mL}, 2.2$ mmol) was added dropwise to an iced cooled stirred solution of DMAP (42 mg, $0.34 \mathrm{mmol})$, triethylamine $(0.7 \mathrm{~mL})$ and 1-(3-hydroxy-azetidin-1-yl)-ethanone $4(206 \mathrm{mg}, 1.79 \mathrm{mmol})^{40}$ in methylene chloride $(10 \mathrm{~mL})$ and after 1 hour the ice bath was removed and the reaction was 
stirred for 24 hours at room temperature. The solution was poured into ice-cold water $(10 \mathrm{~mL})$, extracted with methylene chloride $(3 \times 10 \mathrm{~mL})$, the combined extracts washed with cold $10 \% \mathrm{HCl}$ $(10 \mathrm{~mL})$, water $(10 \mathrm{~mL})$, dried over magnesium sulfate and concentrated under reduced pressure. For characterisation the crude mixture was purified by preparative tlc (eluent; chloroform:methanol 95:5, $\mathrm{R}_{\mathrm{f}}: 0.13$ ) to give the titled compound (224 $\mathrm{mg}, 65 \%$ ) as an oil. The crude product was sufficiently pure for use in the next step. HRMS (EI): $\mathrm{C}_{6} \mathrm{H}_{12} \mathrm{NO}_{4} \mathrm{~S}$ requires $\mathrm{M}^{+1}$ 194.0487, found $\mathrm{M}^{+1} 194.0490 ; \mathrm{v}_{\max }(\mathrm{KBr}) / \mathrm{cm}^{-1} 2938,1651,1457,1355,1171 ;{ }^{1} \mathrm{H} \mathrm{NMR}$ $\left(500.1 \mathrm{MHz}, \mathrm{CDCl}_{3}\right) \delta(\mathrm{ppm}) 1.90(3 \mathrm{H}, \mathrm{s}), 3.09(3 \mathrm{H}, \mathrm{s}), 4.16(1 \mathrm{H}, \mathrm{dd}, \mathrm{J}=11.2,3.7 \mathrm{~Hz}), 4.34$ $(1 \mathrm{H}, \mathrm{dd}, \mathrm{J}=9.9,3.7 \mathrm{~Hz}), 4.39(1 \mathrm{H}, \mathrm{dd}, \mathrm{J}=11.2,6.9 \mathrm{~Hz}), 4.50(1 \mathrm{H}, \mathrm{dd}, \mathrm{J}=9.9,6.9 \mathrm{~Hz}), 5.29$ $(1 \mathrm{H}, \mathrm{tt}, \mathrm{J}=6.9,3.7 \mathrm{~Hz}) ;{ }^{13} \mathrm{C} \mathrm{NMR}\left(125.8 \mathrm{MHz}, \mathrm{CDCl}_{3}\right) \delta(\mathrm{ppm}) 18.9,38.4,55.0,57.7,66.5$, 170.6; MS (EI), $m / z(\%), 194\left(\mathrm{M}^{+1}, 4\right), 193\left(\mathrm{M}^{+}, 2\right), 114$ (18), 97 (7), 72 (67), 43 (100).

$\mathrm{N}$-Acetyl-2-azetine (6). Potassium tert-butoxide $(64.0 \mathrm{~g}, 571 \mathrm{mmol})$ in warm, anhydrous, tertbutanol $(500 \mathrm{~mL})$ was added, over a period 20 minutes to a solution of methanesulfonic acid 1acetyl-azetidin-3-yl ester $5(60.0 \mathrm{~g}, 309 \mathrm{mmol})$ in anhydrous tert-butanol $(200 \mathrm{~mL})$, under nitrogen, at $55^{\circ} \mathrm{C}$ and the resulting mixture was stirred for 3 hours. The solvent was removed in vacuo and the residue was vigorously stirred with diethyl ether, $(250 \mathrm{~mL})$, for 20 minutes, filtered, and concentrated in vacuo to yield $16.8 \mathrm{~g}$ of an oily substance. The solid particulate was then suspended in water $(100 \mathrm{~mL})$ and the product was extracted with methylene chloride $(50$ $\mathrm{mL} \times 3$ ). The resultant emulsion was filtered through Hyflo-Supercel and the solvent was removed under reduced pressure to yield $3.5 \mathrm{~g}$ of an oily substance. Both oily residues were combined, dissolved in methylene chloride $(70 \mathrm{~mL})$, washed with water $(30 \mathrm{~mL})$, dried over magnesium sulfate, and concentrated to give a solid which was recrystallised from hexane to yield the titled compound (14.6 g, 49\%) as colorourless needles, mp $44-46{ }^{\circ} \mathrm{C}$. HRMS (EI) $\mathrm{C}_{5} \mathrm{H}_{7} \mathrm{NO}$ requires $\mathrm{M}^{+} 97.0528$, found $\mathrm{M}^{+} 97.0530 ; \mathrm{v}_{\max }(\mathrm{KBr}) / \mathrm{cm}^{-1} 2964,1652,1418 ;{ }^{1} \mathrm{H}$ NMR $\left(500.1 \mathrm{MHz}, \mathrm{CDCl}_{3}\right) \delta(\mathrm{ppm})\left(\right.$ mixture of rotamers at $\left.25^{\circ} \mathrm{C}\right) 1.97$ and $2.05(3 \mathrm{H}, \mathrm{bs}), 4.44$ and $4.53(2 \mathrm{H}, \mathrm{b}), 5.70$ and $5.72(1 \mathrm{H}, \mathrm{b}), 6.62$ and $6.92(1 \mathrm{H}, \mathrm{b}){ }^{13} \mathrm{C} \mathrm{NMR}\left(125.8 \mathrm{MHz}, \mathrm{CDCl}_{3}\right) \delta$ (ppm) (mixture of rotamers at $25^{\circ} \mathrm{C}$ ) 18.4 and 19.3, 56.5 and 58.8, 113.1 and 113.3, 137.2 and 137.6, 164.4 and 164.8. MS (EI), $m / z(\%), 97\left(\mathrm{M}^{+} 55\right), 55$ (68), 43 (100), 28 (40).

\section{General procedure for multi component coupling reaction}

$\mathrm{N}$-(2-Phenyl-4-phenylamino-1,2,3,4-tetrahydro-quinolin-3-ylmethyl) acetamide 12a and 13a A solution of phenyl-[1-phenyl-meth-(E)-ylidene]-amine 7a (111 mg, $0.61 \mathrm{mmol}), \mathrm{N}$-acetyl-2azetine 6 (59 mg, $0.61 \mathrm{mmol})$, aniline $(68 \mathrm{mg}, 0.73 \mathrm{mmol})$ and yttrium triflate $(10 \mathrm{mg}, 3.0 \mathrm{~mol} \%)$ in anhydrous acetonitrile $(10 \mathrm{~mL})$ was stirred at room temperature under nitrogen for 24 hours. The solvent was removed under reduced pressure, the residue dissolved in methylene chloride $(30 \mathrm{~mL})$, washed with aq. saturated sodium bicarbonate solution $(10 \mathrm{~mL})$, water $(10 \mathrm{~mL})$, dried over magnesium sulfate and concentrated in vacuo. Purification by flash chromatography (eluent; hexane:ethyl ethanoate 1:1, $\mathrm{R}_{\mathrm{f}}$ : 0.36) afforded 12a (206 $\left.\mathrm{mg}, 91 \%\right)$ as a cream foam $\mathrm{mp}$ 76-78 ${ }^{\circ} \mathrm{C}$; HRMS (FAB): $\mathrm{C}_{24} \mathrm{H}_{25} \mathrm{~N}_{3} \mathrm{O}$ requires $\mathrm{M}^{+} 371.1998$, found $\mathrm{M}^{+} 371.1988 ; \mathrm{v}_{\max }(\mathrm{KBr}) / \mathrm{cm}^{-}$

${ }^{1} 3397,3206,1652,1498,1310,750,696 ;{ }^{1} \mathrm{H}$ NMR (500.1 MHz, $\left.\mathrm{CDCl}_{3}\right) \delta(\mathrm{ppm}) 1.55(3 \mathrm{H}, \mathrm{s})$, 
2.40 (1H, br m), $3.11(1 \mathrm{H}$, ddd, $\mathrm{J}=14.0,6.3,5.3 \mathrm{~Hz}), 3.30(1 \mathrm{H}$, ddd $\mathrm{J}=14.0,6.5,5.3 \mathrm{~Hz}), 4.03$ $(1 \mathrm{H}, \mathrm{br}), 4.21(1 \mathrm{H}, \mathrm{s}), 4.45(1 \mathrm{H}, \mathrm{d}, \mathrm{J}=1.3 \mathrm{~Hz}), 4.71(1 \mathrm{H}, \mathrm{d}, \mathrm{J}=2.8 \mathrm{~Hz}), 5.13(1 \mathrm{H}$, br t, J $=5.3$ $\mathrm{Hz}), 6.70(1 \mathrm{H}, \mathrm{d}, \mathrm{J}=8.2 \mathrm{~Hz}), 6.72(2 \mathrm{H}, \mathrm{d}, \mathrm{J}=7.7 \mathrm{~Hz}), 6.77(2 \mathrm{H}$, overlap m), $7.14(1 \mathrm{H}, \mathrm{td}, \mathrm{J}=$ 8.1, $1.5 \mathrm{~Hz}), 7.23\left(3 \mathrm{H}\right.$, overlap m), $7.29(1 \mathrm{H}, \mathrm{m}), 7.37\left(4 \mathrm{H}\right.$, overlap m); ${ }^{13} \mathrm{C} \mathrm{NMR}(125.8 \mathrm{MHz}$, $\left.\mathrm{CDCl}_{3}\right) \delta(\mathrm{ppm}) 22.8,37.1,43.5,53.5,53.7,112.9(2 \mathrm{C}), 115.2,117.7,118.9,120.6,126.4(2 \mathrm{C})$, 127.6, 128.9(3C), 129.6(2C), 131.6, 141.6, 144.2, 146.2, 169.9; MS (EI), m/z (\%), $371\left(\mathrm{M}^{+}, 7\right)$, 279 (51), 277 (11), 220 (62), 206 (100).

On repetition of this experiment, and washing the silica with 5\% triethylamine solution prior to chromatography, an additional products 13a (9 $\mathrm{mg}, 3 \%)$ was obtained as a colorourless powder, mp 160-162 ${ }^{\circ} \mathrm{C}$, ( $\mathrm{R}_{\mathrm{f}}$ : 0.53 hexane:ethyl ethanoate 1:1) HRMS (EI): $\mathrm{C}_{24} \mathrm{H}_{25} \mathrm{~N}_{3} \mathrm{O}$ requires $\mathrm{M}^{+}$ 371.1998, found $\mathrm{M}^{+}$371.1983; $\mathrm{U}_{\max }(\mathrm{KBr}) / \mathrm{cm}^{-1} 3373,3315,1641,1253,752,696$; ${ }^{1} \mathrm{H}$ NMR $\left(500.1 \mathrm{MHz}, \mathrm{CDCl}_{3}\right) \delta(\mathrm{ppm}) 1.35(3 \mathrm{H}, \mathrm{s}), 1.60(1 \mathrm{H}, \mathrm{br}), 2.42(1 \mathrm{H}, \mathrm{m}), 2.84(1 \mathrm{H}, \mathrm{ddd}, \mathrm{J}=14.7$, 3.4, 3.4 Hz), 3.69 (1H, ddd, J = 14.7, 9.4, $4.5 \mathrm{~Hz}), 4.15(1 \mathrm{H}, \mathrm{s}),, 5.00(1 \mathrm{H}, \mathrm{d}, \mathrm{J}=2.5 \mathrm{~Hz}), 5.26$ $(1 \mathrm{H}, \mathrm{br}), 5.76(1 \mathrm{H}, \mathrm{br}), 6.64(1 \mathrm{H}, \mathrm{d}, \mathrm{J}=7.9 \mathrm{~Hz}), 6.70(1 \mathrm{H}, \mathrm{t}, \mathrm{J}=7.3 \mathrm{~Hz}), 6.78(1 \mathrm{H}, \mathrm{t}, \mathrm{J}=7.5 \mathrm{~Hz})$, $6.81(2 \mathrm{H}, \mathrm{d}, \mathrm{J}=7.7 \mathrm{~Hz}), 7.06(1 \mathrm{H}, \mathrm{t}, \mathrm{J}=7.5 \mathrm{~Hz}), 7.20(2 \mathrm{H}, \mathrm{t}, \mathrm{J}=7.9 \mathrm{~Hz}), 7.33(1 \mathrm{H}, \mathrm{d}, \mathrm{J}=7.0$ $\mathrm{Hz}), 7.40-7.45\left(4 \mathrm{H}\right.$, overlapping m), $7.49(1 \mathrm{H}, \mathrm{d}, \mathrm{J}=7.6 \mathrm{~Hz}) ;{ }^{13} \mathrm{C} \mathrm{NMR}\left(125.8 \mathrm{MHz}, \mathrm{CDCl}_{3}\right) \delta$ (ppm) 21.4, 34.7, 41.7, 53.2, 59.7, 113.8(2C), 114.9, 117.7, 119.9, 124.2, 126.4(2C), 127.4, 128.2, 128.3, 129.3(2C), 129.9(2C), 141.6, 144.5, 148.5, 171.5; MS (EI), $m / z(\%), 371\left(\mathrm{M}^{+}, 2\right)$, 280 (1), 236 (3), 206 (10), 121 (96), 93 (100), 57 (94), 43 (71).

\section{$N$-[6-Methoxy-4-(4-methoxy-phenylamino)-2-phenyl-1,2,3,4-tetrahydro-quinolin-3}

ylmethyl]-acetamide (12b). (4-Methoxy-phenyl)-[1-phenyl-meth-(E)-ylidene]-amine $7 \mathbf{b}$ (124 $\mathrm{mg}, 0.55 \mathrm{mmol}$ ), $N$-acetyl-2-azetine (51 mg, $0.53 \mathrm{mmol})$, 4-methoxyaniline (68 $\mathrm{mg}, 0.55 \mathrm{mmol}$ ) and yttrium triflate $(9 \mathrm{mg}, 3.0 \mathrm{~mol} \%)$ in acetonitrile $(2 \mathrm{~mL})$ were reacted according to general procedure for 48 hours to give the titled compound $\mathbf{1 2 b}(193 \mathrm{mg}, 85 \%)$ as a cream coloured powder mp 91-94 ${ }^{\circ} \mathrm{C},\left(\mathrm{R}_{\mathrm{f}} 0.19\right.$, ethyl ethanoate:hexane 1:1,); HRMS (FAB): $\mathrm{C}_{26} \mathrm{H}_{29} \mathrm{~N}_{3} \mathrm{O}_{3}$ requires $\mathrm{M}^{+}$431.2209, found $\mathrm{M}^{+} 431.2193 ; \mathrm{v}_{\max }(\mathrm{KBr}) / \mathrm{cm}^{-1} 3312,1655,1235,1036,820,703 ;{ }^{1} \mathrm{H} \mathrm{NMR}$ $\left(500.1 \mathrm{MHz}, \mathrm{CDCl}_{3}\right) \delta(\mathrm{ppm}) 1.54(3 \mathrm{H}, \mathrm{s}), 2.35(1 \mathrm{H}, \mathrm{m}), 3.09(1 \mathrm{H}, \mathrm{ddd}, \mathrm{J}=14.2,5.8,4.4 \mathrm{~Hz})$, $3.33(1 \mathrm{H}, \mathrm{dt}, \mathrm{J}=14.2,6.3 \mathrm{~Hz}), 3.72(3 \mathrm{H}, \mathrm{s}), 3.77(3 \mathrm{H}, \mathrm{s}), 4.00(1 \mathrm{H}, \mathrm{br}), 4.35(1 \mathrm{H}, \mathrm{s}), 4.65(1 \mathrm{H}, \mathrm{d}$, $\mathrm{J}=2.7 \mathrm{~Hz}$ ), $5.25(1 \mathrm{H}, \mathrm{br}), 6.65-6.70$ (3H, overlapping $\mathrm{m}), 6.68-6.85$ (4H, overlapping $\mathrm{m})$, 7.297.50 (5H, overlapping m); ${ }^{13} \mathrm{C}$ NMR (125.8 $\left.\mathrm{MHz} \mathrm{CDCl}_{3}\right) \delta$ (ppm) 21.8, 36.2, 42.5, 53.0, 53.5, 54.80, 54.83, 113.3(2C), 113.9, 114.2(2C), 115.4, 115.5, 120.8, 125.3(2C), 126.5, 127.6(2C), 137.2, 139.5, 140.9, 151.3, 151.9, 168.9; MS (EI), m/z (\%), $431\left(\mathrm{M}^{+}, 17\right), 309$ (22), 250 (65), 236 (100).

$\mathrm{N}$-[Methyl-2-(4-nitro-phenyl)-4-p-tolylamino-1,2,3,4-tetrahydro-quinolin-3-ylmethyl]acetamide (12c). [1-(4-Nitro-phenyl)-meth-(E)-ylidene]-p-tolyl-amine 7c (130 mg, $0.54 \mathrm{mmol})$, $N$-acetyl-2-azetine $6(51 \mathrm{mg}, 0.53 \mathrm{mmol})$, 4-methylaniline $(58 \mathrm{mg}, 0.54 \mathrm{mmol})$ and yttrium triflate $(11 \mathrm{mg}, 4.0 \mathrm{~mol} \%)$ in acetonitrile $(10 \mathrm{~mL})$ were reacted under the general procedure for 40 hours to give the titled compound 12c (66 mg, 28\%) as a yellow solid mp 204-206 ${ }^{\circ} \mathrm{C}$; HRMS (FAB): $\mathrm{C}_{26} \mathrm{H}_{28} \mathrm{~N}_{4} \mathrm{O}_{3}$ requires $\mathrm{M}^{+}$444.2161, found $\mathrm{M}^{+} 444.2156$; $\mathrm{v}_{\max }(\mathrm{KBr}) / \mathrm{cm}^{-1} 3369,3265$, 
1650, 1514, 1346; ${ }^{1} \mathrm{H}$ NMR (500.1 MHz, $\left.\mathrm{CDCl}_{3}\right) \delta(\mathrm{ppm}) 1.70(3 \mathrm{H}, \mathrm{s}), 2.24(3 \mathrm{H}, \mathrm{s}), 2.27(3 \mathrm{H})$, 2.47 (1H, br m), $2.93(1 \mathrm{H}, \mathrm{dt}, \mathrm{J}=14.2,6.1 \mathrm{~Hz}), 3.34(1 \mathrm{H}, \mathrm{ddd}, \mathrm{J}=14.2,6.5,5.2 \mathrm{~Hz}), 4.08(1 \mathrm{H}$, br), $4.42(1 \mathrm{H}, \mathrm{d}, \mathrm{J}=1.4 \mathrm{~Hz}), 4.79(1 \mathrm{H}, \mathrm{d}, \mathrm{J}=2.6 \mathrm{~Hz}), 5.40(1 \mathrm{H}, \mathrm{br}), 6.61(2 \mathrm{H}, \mathrm{d}, \mathrm{J}=8.4 \mathrm{~Hz})$, $6.67(1 \mathrm{H}, \mathrm{d}, \mathrm{J}=8.2 \mathrm{~Hz}), 6.99(1 \mathrm{H}, \mathrm{d}, \mathrm{J}=8.2 \mathrm{~Hz}), 7.04(2 \mathrm{H}, \mathrm{d}, \mathrm{J}=8.3 \mathrm{~Hz}), 7.07(1 \mathrm{H}, \mathrm{s}), 7.57$ $(2 \mathrm{H}, \mathrm{d}, \mathrm{J}=8.8 \mathrm{~Hz}), 8.22(2 \mathrm{H}, \mathrm{d}, \mathrm{J}=8.8 \mathrm{~Hz}) ;{ }^{13} \mathrm{C} \mathrm{NMR}\left(125.8 \mathrm{M} \mathrm{Hz}, \mathrm{CDCl}_{3}\right) \delta(\mathrm{ppm}) 20.8,20.9$, 23.2, 37.2, 43.6, 53.6, 54.3, 113.3(2C), 116.1, 121.2, 124.2(2C), 127.7, 127.8(2C), 129.4, 130.1, 130.2(2C), 132.1, 141.6, 144.2, 147.6, 149.8, 170.5; MS (FAB), $m / z(\%), 444\left(\mathrm{M}^{+}, 15\right), 338(44)$, 279 (60), 265 (100).

A heavy colorourless precipitate formed during the course of this reaction. The solid was filtered and the solid material (70 mg, 30\%) was identified as 8c by NMR spectroscopy. The instability of this material precluded further characterization and this was used for an attempted cross over experiment.

$\mathrm{N}$-[2-(4-Methoxy-phenyl)-4-phenylamino-1,2,3,4-tetrahydro-quinolin-3-ylmethyl]acetamide (12d). [1-(4-Methoxy-phenyl)-meth-(E)-ylidene]-phenyl-amine 7d (150 mg, 0.71 mmol), $N$-acetyl-2-azetine $6(67 \mathrm{mg}, 0.69 \mathrm{mmol})$, aniline $(68 \mathrm{mg}, 0.73 \mathrm{mmol})$ and yttrium triflate (12 $\mathrm{mg}, 3.2 \mathrm{~mol} \%)$ in anhydrous acetonitrile $(10 \mathrm{~mL})$ were reacted according to general procedure for 30 hours afforded the titled compound 12d (268 mg, 97\%) as a cream powder, $\mathrm{mp}$ 91-94 ${ }^{\circ} \mathrm{C}$ ( $\mathrm{R}_{\mathrm{f}}$ : 0.25 , ethyl ethanoate:hexane, 1:1); HRMS (EI): $\mathrm{C}_{25} \mathrm{H}_{27} \mathrm{~N}_{3} \mathrm{O}_{2}$ requires $\mathrm{M}^{+}$ 401.2103, found $\mathrm{M}^{+}$401.2088; $\mathrm{v}_{\max }(\mathrm{KBr}) / \mathrm{cm}^{-1} 3408,3290,1649,1311,1251,752 ;{ }^{1} \mathrm{H} \mathrm{NMR}$ $\left(500.1 \mathrm{MHz}, \mathrm{CDCl}_{3}\right) \delta(\mathrm{ppm}) 1.59(3 \mathrm{H}, \mathrm{s}), 2.33(1 \mathrm{H}, \mathrm{br}), 3.07(1 \mathrm{H}, \mathrm{ddd}, \mathrm{J}=14.2,5.5,1.1 \mathrm{~Hz})$, $3.33(1 \mathrm{H}, \mathrm{ddd}, \mathrm{J}=14.2,8.1,6.0 \mathrm{~Hz}), 3.79(3 \mathrm{H}, \mathrm{s}), 3.98(1 \mathrm{H}, \mathrm{br}), 4.18(1 \mathrm{H}, \mathrm{s}), 4.43(1 \mathrm{H}, \mathrm{d}, \mathrm{J}=$ $1.4 \mathrm{~Hz}), 4.65(1 \mathrm{H}, \mathrm{d}, \mathrm{J}=2.8 \mathrm{~Hz}), 5.25(1 \mathrm{H}, \mathrm{br}), 6.69(3 \mathrm{H}$, overlap m), $6.74(2 \mathrm{H}, \mathrm{q}, \mathrm{J}=7.4 \mathrm{~Hz})$, $6.89(2 \mathrm{H}, \mathrm{d}, \mathrm{J}=8.7 \mathrm{~Hz}), 7.13(1 \mathrm{H}, \mathrm{t}, \mathrm{J}=7.6 \mathrm{~Hz}), 7.21(3 \mathrm{H}$, overlapping $\mathrm{m}), 7.29(2 \mathrm{H}, \mathrm{d}, \mathrm{J}=8.7$ $\mathrm{Hz}) ;{ }^{13} \mathrm{C}$ NMR (125.8 MHz, $\left.\mathrm{CDCl}_{3}\right) \delta(\mathrm{ppm}) 21.1,36.1,42.3,52.2,52.4,54.4,111.9(2 \mathrm{C})$, 113.2(2C), 114.2, 116.7, 117.9, 119.6, 126.5(2C), 127.8, 128.5(2C), 130.5, 132.3, 143.3, 145.2, 158.0, 168.9; MS (EI), m/z (\%), $401\left(\mathrm{M}^{+}, 1\right), 329$ (1), 248 (95), 236 (100), 93 (97), 57 (67), 43 (70), 28 (93).

3-(Acetylamino-methyl)-4-phenylamino-1,2,3,4-tetrahydro-quinoline-2-carboxylic acid ethyl ester (12e). [(E)-Phenylimino]-acetic acid ethyl ester 7e (50 mg, $0.28 \mathrm{mmol}), \mathrm{N}$-acetyl-2azetine 6 (21 mg, $0.22 \mathrm{mmol})$, aniline $(23 \mathrm{mg}, 0.25 \mathrm{mmol})$ and yttrium triflate $(3 \mathrm{mg}, 3 \mathrm{~mol} \%)$ in anhydrous acetonitrile $(5 \mathrm{~mL})$ were reacted according to general procedure to give the titled compound 12e (77 mg, 97\%) as a cream coloured powder mp 62-64 ${ }^{\circ} \mathrm{C}$, ( $\mathrm{R}_{\mathrm{f}}$ : 0.41 , ethyl ethanoate:hexane 7:3,); HRMS (EI): $\mathrm{C}_{21} \mathrm{H}_{25} \mathrm{~N}_{3} \mathrm{O}_{3}$ requires $\mathrm{M}^{+}$367.1896, found $\mathrm{M}^{+}$367.1883; $v_{\max }(\mathrm{KBr}) / \mathrm{cm}^{-1} 3398,3314,1731,1657,1602,1499,1297,751,694 ;{ }^{1} \mathrm{H}$ NMR $(500.1 \mathrm{MHz}$, $\left.\mathrm{CDCl}_{3}\right) \delta(\mathrm{ppm}) 1.28(3 \mathrm{H}, \mathrm{t}, \mathrm{J}=7.1 \mathrm{~Hz}), 1.82(3 \mathrm{H}, \mathrm{s}), 2.86(1 \mathrm{H}, \mathrm{m}), 3.17(2 \mathrm{H}, \mathrm{m}), 3.84(1 \mathrm{H}, \mathrm{br})$, $4.19(1 \mathrm{H}, \mathrm{d}, \mathrm{J}=2.8 \mathrm{~Hz}), 4.23(2 \mathrm{H}, \mathrm{m}), 4.31(1 \mathrm{H}, \mathrm{d}, \mathrm{J}=1.4 \mathrm{~Hz}), 4.62(1 \mathrm{H}, \mathrm{br} \mathrm{s}), 5.80(1 \mathrm{H}, \mathrm{br} \mathrm{t}, \mathrm{J}$ $=5.8 \mathrm{~Hz}), 6.64-6.76(5 \mathrm{H}, \mathrm{m}), 7.10-7.17(3 \mathrm{H}, \mathrm{m}), 7.23(1 \mathrm{H}, \mathrm{d}, \mathrm{J}=7.4 \mathrm{~Hz}) ;{ }^{13} \mathrm{C} \mathrm{NMR}(125.8$ $\left.\mathrm{MHz}, \mathrm{CDCl}_{3}\right) \delta$ (ppm) 14.6, 23.3, 38.3, 38.8, 52.6, 53.5, 62.1, 113.1(2C), 115.2, 118.3, 118.9, 119.7, 129.6, 129.9(2C), 131.8, 142.8, 146.7, 170.6, 173.7; MS (EI), m/z (\%), $367\left(\mathrm{M}^{+}, 1\right), 309$ (4), 272 (51), 202 (95), 183 (75), 157 (87), 143 (86), 130 (72), 93 (70), 43 (100). 


\section{$N$-[6-Methoxy-4-(4-methoxy-phenylamino)-2-pent-1-enyl-1,2,3,4-tetra-hydro-quinolin-3-} ylmethyl]-acetamide (12f) [(E)-Hex-2-en-(E)-ylidene]-phenyl-amine 7f (110 mg, $0.54 \mathrm{mmol})$, $N$-acetyl-2-azetine 6 (50 mg, $0.51 \mathrm{mmol})$, 4-methoxyaniline $(70 \mathrm{mg}, 0.57 \mathrm{mmol})$ and yttrium triflate $(11 \mathrm{mg}, 4.0 \mathrm{~mol} \%)$ in anhydrous acetonitrile $(10 \mathrm{~mL})$ were reacted according to the general procedure for $30 \mathrm{hr}$ to give the titled compound $\mathbf{1 2 f}(65 \mathrm{mg}, 30 \%)$ as an oil, ( $\mathrm{R}_{\mathrm{f}}$ : 0.14 , hexane:ethyl ethanoate 1:1); HRMS (EI): $\mathrm{C}_{25} \mathrm{H}_{33} \mathrm{~N}_{3} \mathrm{O}_{3}$ requires $\mathrm{M}^{+} 423.2523$, found $\mathrm{M}^{+} 423.2518$ $U_{\max }(\mathrm{KBr}) / \mathrm{cm}^{-1} 3325,1652,1510,1243,1037,820,733 ;{ }^{1} \mathrm{H} \mathrm{NMR}\left(500.1 \mathrm{MHz}, \mathrm{CDCl}_{3}\right) \delta(\mathrm{ppm})$ $0.91(3 \mathrm{H}, \mathrm{t}, \mathrm{J}=7.4 \mathrm{~Hz}), 1.41(2 \mathrm{H}, \mathrm{m}), 1.75(3 \mathrm{H}, \mathrm{s}), 2.05(2 \mathrm{H}, \mathrm{m}), 2.20(1 \mathrm{H}, \mathrm{m}), 3.13(1 \mathrm{H}, \mathrm{dt}, \mathrm{J}=$ 14.1, $5.8 \mathrm{~Hz}), 3.65(1 \mathrm{H}, \mathrm{m}), 3.70(3 \mathrm{H}, \mathrm{s}), 3.76(3 \mathrm{H}, \mathrm{s}), 3.97(1 \mathrm{H}, \mathrm{m}), 4.32(1 \mathrm{H}, \mathrm{d}, \mathrm{J}=2.2 \mathrm{~Hz})$, $5.59(1 \mathrm{H}, \mathrm{dd}, \mathrm{J}=15.5,6.1 \mathrm{~Hz}), 5.74(1 \mathrm{H}, \mathrm{dtd}, \mathrm{J}=15.5,6.4,1.3 \mathrm{~Hz}), 5.93(1 \mathrm{H}, \mathrm{br} \mathrm{t}, \mathrm{J}=5.8 \mathrm{~Hz})$, $6.57(1 \mathrm{H}, \mathrm{d}, \mathrm{J}=8.7 \mathrm{~Hz}), 6.63(2 \mathrm{H}, \mathrm{d}, \mathrm{J}=8.9 \mathrm{~Hz}), 6.73(1 \mathrm{H}, \mathrm{dd}, \mathrm{J}=8.7,2.9 \mathrm{~Hz}), 6.77(1 \mathrm{H}, \mathrm{d}, \mathrm{J}=$ $2.9 \mathrm{~Hz}), 6.81(2 \mathrm{H}, \mathrm{d}, \mathrm{J}=8.9 \mathrm{~Hz}) ;{ }^{13} \mathrm{C} \mathrm{NMR}\left(125.8 \mathrm{MHz}, \mathrm{CDCl}_{3}\right) \delta(\mathrm{ppm}) 13.7,22.4,29.7,34.6$, $38.1,40.9$, 52.2, 53.9, 55.8, 55.9, 114.1(2C), 114.8, 115.2 (2C), 116.1, 116.2, 122.2, 129.7, 132.4, 138.1, 140.7, 152.2, 152.8, 170.2; MS (EI), m/z (\%), $423\left(\mathrm{M}^{+}, 3\right), 302$ (18), 299 (24), 242 (75), 228 (100).

\section{$\mathrm{N}$-[6-Methyl-2-(4-nitro-phenyl)-4-phenylamino-1,2,3,4-tetrahydroquinolin-3-ylmethyl]-}

acetamide (12g). A suspension of 1-[7-methyl-3-(4-nitro-phenyl)-2a,3,4,8b-tetrahydro-2H-1,4diaza-cyclobuta[a]naphthalen-1-yl]-ethanone $8 \mathbf{c}(27 \mathrm{mg}, 0.08 \mathrm{mmol})$, aniline (8 $\mathrm{mg}, 0.09 \mathrm{mmol})$ and yttrium triflate $(3.5 \mathrm{mg}, 8 \mathrm{~mol} \%)$ in anhydrous acetonitrile $(5 \mathrm{~mL})$ was reacted according to general procedure for 48 hours to give to give the titled compound (22.6 $\mathrm{mg}, 66 \%$ ) as a yellow solid mp 255-258 ${ }^{\circ} \mathrm{C},\left(\mathrm{R}_{\mathrm{f}}\right.$ : 0.38; ethyl ethanoate:hexane 1:1); MS (EI): $\mathrm{C}_{25} \mathrm{H}_{24} \mathrm{~N}_{4} \mathrm{O}_{3}$ requires $\mathrm{M}^{+}$ 430.2006, found $\mathrm{M}^{+} 430.1894 \mathrm{v}_{\max }(\mathrm{KBr}) / \mathrm{cm}^{-1} 3369,3270,1650,1507,1346,748$; ${ }^{1} \mathrm{H}$ NMR $\left(500.1 \mathrm{MHz}, \mathrm{CDCl}_{3}\right) \delta(\mathrm{ppm}) 1.68(3 \mathrm{H}, \mathrm{s}), 2.25(3 \mathrm{H}, \mathrm{s}), 2.48(1 \mathrm{H}, \mathrm{m}), 2.95(1 \mathrm{H}, \mathrm{dt}, \mathrm{J}=14.3,6.0$, $\mathrm{Hz}), 3.35(1 \mathrm{H}, \mathrm{ddd}, \mathrm{J}=14.3,5.9,5.7 \mathrm{~Hz}), 4.09(1 \mathrm{H}, \mathrm{br}), 4.46(1 \mathrm{H}, \mathrm{s}), 4.80(1 \mathrm{H}, \mathrm{d}, \mathrm{J}=2.6 \mathrm{~Hz})$, $5.38(1 \mathrm{H}, \mathrm{br}), 6.68(3 \mathrm{H}$, overlap $\mathrm{m}), 6.78(1 \mathrm{H}, \mathrm{t}, \mathrm{J}=7.4), 6.99(1 \mathrm{H}, \mathrm{dd}, \mathrm{J}=8.2,1.7 \mathrm{~Hz}), 7.08$ $(1 \mathrm{H}, \mathrm{s}), 7.13(2 \mathrm{H}, \mathrm{t}, \mathrm{J}=7.4), 7.58(2 \mathrm{H}, \mathrm{d}, \mathrm{J}=8.5 \mathrm{~Hz}), 8.22(2 \mathrm{H}, \mathrm{d}, \mathrm{J}=8.5 \mathrm{~Hz}) ;{ }^{13} \mathrm{C} \mathrm{NMR}(125.8$ $\left.\mathrm{MHz}, \mathrm{CDCl}_{3}\right) \delta(\mathrm{ppm}) 19.4,21.8,35.8,42.2,51.9,52.9,111.8(2 \mathrm{C}), 114.7,117.0,119.6$, 122.9(2C), 126.4(2C), 128.1, 128.6(2C), 128.9, 130.8, 140.3, 145.1, 146.3, 148.3, 169.1; MS (ES), $m / z(\%), 430\left(\mathrm{M}^{+}, 12\right) 338$ (43), 279 (66), 265 (100).

$\mathrm{N}$-(6-Methoxy-2-phenyl-quinolin-3-yl-methyl)-acetamide (11b). A solution of $N$-[6-methoxy4-(4-methoxy-phenylamino)-2-phenyl-1,2,3,4-tetrahydro-quinolin-3-ylmethyl]-acetamide $\mathbf{1 2 b}$ (30 $\mathrm{mg}, 0.07 \mathrm{mmol})$ and acetic acid $(1.5 \mathrm{~mL}, 26.2 \mathrm{mmol})$ in methylene chloride $(7 \mathrm{~mL})$ was stirred at room temperature for 48 hours. The solution was washed with saturated aq. sodium bicarbonate $(10 \mathrm{~mL})$, water $(10 \mathrm{~mL})$, dried over magnesium sulfate, concentrated and purified by flash chromatography (eluent; ethyl ethanoate:hexane 7:3, $\mathrm{R}_{\mathrm{f}}: 0.45$ ) to give the titled compound 11b, (15 mg, 71\%) as a colorourless solid. mp 216-217 ${ }^{\circ} \mathrm{C}$. HRMS (EI): $\mathrm{C}_{19} \mathrm{H}_{18} \mathrm{~N}_{2} \mathrm{O}_{2}$ requires $\mathrm{M}^{+}$ 306.1368, found $\mathrm{M}^{+} 306.1384$; $\mathrm{v}_{\max }(\mathrm{KBr}) / \mathrm{cm}^{-1} 3271,1658,1551,1226,762,703$; ${ }^{1} \mathrm{H} \mathrm{NMR}$ $\left(500.1 \mathrm{MHz}, \mathrm{CDCl}_{3}\right) \delta(\mathrm{ppm}) 1.95(3 \mathrm{H}, \mathrm{s}), 3.94(3 \mathrm{H}, \mathrm{s}), 4.55(2 \mathrm{H}, \mathrm{d}, \mathrm{J}=6.0 \mathrm{~Hz}), 5.73(1 \mathrm{H}, \mathrm{br})$, $7.09(1 \mathrm{H}, \mathrm{d}, \mathrm{J}=2.8 \mathrm{~Hz}), 7.36(1 \mathrm{H}, \mathrm{dd}, \mathrm{J}=9.2,2.8 \mathrm{~Hz}), 7.44-7.53(5 \mathrm{H}), 8.01(1 \mathrm{H}, \mathrm{d}, \mathrm{J}=9.2 \mathrm{~Hz})$, $8.09(1 \mathrm{H}, \mathrm{s}) ;{ }^{13} \mathrm{C}$ NMR $\left(125.8 \mathrm{MHz}, \mathrm{CDCl}_{3}\right) \delta(\mathrm{ppm}) 23.2,41.4,55.6,104.8,122.6,128.4,128.5$, 
128.65(2C), 128.74(2C), 129.9, 130.7, 135.1, 140.1, 143.2, 156.9, 158.1, 169.9; MS (EI), $m / z$ (\%), $307\left(\mathrm{M}^{+}+1,5\right), 126(5), 72(100), 58(41)$.

$\mathrm{N}$-[6-Methyl-2-(nitro-phenyl)-quinolin-3-ylmethyl]-acetamide (11c). A solution of $\mathrm{N}$ [methyl-2-(4-nitro-phenyl)-4-p-tolylamino-1,2,3,4-tetrahydro-quinolin-3-ylmethyl]-acetamide

12c (120 mg, $0.27 \mathrm{mmol})$ and yttrium triflate $(7 \mathrm{mg}, 5.0 \mathrm{~mol} \%)$ in acetonitrile $(7 \mathrm{~mL})$ was heated to reflux for 4 hours. The solvent was removed, residue dissolved in methylene chloride $(30 \mathrm{~mL})$, washed with saturated aqueous sodium bicarbonate $(10 \mathrm{~mL})$, water $(10 \mathrm{~mL})$, dried over magnesium sulfate, concentrated and purified by flash chromatography (eluent; hexane:ethyl ethanoate 3:7, $\left.\mathrm{R}_{\mathrm{f}}: 0.27\right)$ to give the titled compound $11 \mathrm{c}(71 \mathrm{mg}, 78 \%)$ as a yellow solid mp. 265$266{ }^{\circ} \mathrm{C}$; HRMS (EI): $\mathrm{C}_{19} \mathrm{H}_{17} \mathrm{~N}_{3} \mathrm{O}_{3}$ requires $\mathrm{M}^{+} 335.1270$, found $\mathrm{M}^{+} 335.1255 ; \mathrm{v}_{\max }(\mathrm{KBr}) / \mathrm{cm}^{-1}$ 3303, 2920, 2851, 1639, 1515, 1346; ${ }^{1} \mathrm{H}$ NMR (500.1 MHz, $\left.\mathrm{CDCl}_{3}\right) \delta$ (ppm) $2.00(3 \mathrm{H}, \mathrm{s}), 2.57$ $(3 \mathrm{H}, \mathrm{s}), 4.57(2 \mathrm{H}, \mathrm{d}, \mathrm{J}=5.9 \mathrm{~Hz}), 5.69(1 \mathrm{H}, \mathrm{br}), 7.59(1 \mathrm{H}, \mathrm{dd}, \mathrm{J}=8.6,1.9 \mathrm{~Hz}), 7.63(1 \mathrm{H}, \mathrm{s}), 7.77$ $(2 \mathrm{H}, \mathrm{d}, \mathrm{J}=8.7 \mathrm{~Hz}), 8.00(1 \mathrm{H}, \mathrm{d}, \mathrm{J}=8.6 \mathrm{~Hz}), 8.14(1 \mathrm{H}, \mathrm{s}), 8.36(2 \mathrm{H}, \mathrm{d}, \mathrm{J}=8.7 \mathrm{~Hz}) .{ }^{13} \mathrm{C} \mathrm{NMR}$ (125.8 MHz, d6-DMSO) $\delta$ (ppm) 21.5, 22.8, 39.9 (overlap by DMSO peak), 123.6(2C), 126.5, 127.6, 128.8, 130.7(2C), 131.0, 132.4, 135.3, 137.3, 145.2, 146.6, 147.6, 156.2, 169.6. MS (EI), $\mathrm{m} / z(\%), 335\left(\mathrm{M}^{+}, 2\right), 284$ (15), 242 (18), $111(30), 57$ (100).

3-(Acetylamino-methyl)-quinoline-2-carboxylic acid ethyl ester (11e). A solution of DDQ (203 $\mathrm{mg}, 0.89 \mathrm{mmol})$ in anhydrous acetonitrile $(4 \mathrm{~mL})$ was added drop wise to a solution of 3(acetylamino-methyl)-4-phenylamino-1,2,3,4-tetrahydro-quinoline-2-carboxylic acid ethyl ester 12e $(130 \mathrm{mg}, 0.35 \mathrm{mmol})$ in anhydrous acetonitrile $(3 \mathrm{~mL})$ and the resulting solution was stirred for 2 hours at room temperature followed by the removal of solvent under reduced pressure. The residue was dissolved in methylene chloride $(20 \mathrm{~mL})$, washed with saturated aq. sodium bicarbonate $(10 \mathrm{~mL})$, water $(10 \mathrm{~mL})$, dried over magnesium sulfate and concentrated in vacuo. and purified by flash chromatography (eluent; methylene chloride:methanol 95:5, $\mathrm{R}_{\mathrm{f}}: 0.56$ ) to give the titled compound 11e $(77 \mathrm{mg}, 80 \%)$ as a cream solid $\mathrm{mp} 89-92{ }^{\circ} \mathrm{C}$; HRMS (EI): $\mathrm{C}_{15} \mathrm{H}_{16} \mathrm{~N}_{2} \mathrm{O}_{3}$ requires $\mathrm{M}^{+}$272.1161, found $\mathrm{M}^{+}$272.1155; $\mathrm{U}_{\max }(\mathrm{KBr}) / \mathrm{cm}^{-1} 3299,3081,2977$, 1717, 1644, 1553, 1290, 778, 751; ${ }^{1} \mathrm{H}$ NMR (500.1 MHz, $\left.\mathrm{CDCl}_{3}\right) \delta(\mathrm{ppm}) 1.51(3 \mathrm{H}, \mathrm{t}, \mathrm{J}=7.1$ $\mathrm{Hz}), 1.98(3 \mathrm{H}, \mathrm{s}), 4.56(2 \mathrm{H}, \mathrm{q}, \mathrm{J}=7.1 \mathrm{~Hz}), 4.71(2 \mathrm{H}, \mathrm{d}, \mathrm{J}=6.4 \mathrm{~Hz}), 6.79(1 \mathrm{H}, \mathrm{br}), 7.62(1 \mathrm{H}, \mathrm{t}, \mathrm{J}$ $=8.1 \mathrm{~Hz}), 7.75(1 \mathrm{H}, \mathrm{t}, \mathrm{J}=8.4 \mathrm{~Hz}), 7.84(1 \mathrm{H}, \mathrm{d}, \mathrm{J}=8.1 \mathrm{~Hz}), 8.20(1 \mathrm{H}, \mathrm{d}, \mathrm{J}=8.4 \mathrm{~Hz}), 8.37(1 \mathrm{H}$, s); $\left.{ }^{13} \mathrm{C} \mathrm{NMR} \mathrm{(125.8} \mathrm{MHz,} \mathrm{CDCl}_{3}\right) \delta(\mathrm{ppm})$ 14.3, 23.4, 41.4, 62.5, 127.6, 128.8, 128.9, 130.0, $130.3,131.0,139.3,146.5,148.0,166.9,170.0$; MS (EI), $m / z(\%), 273\left(\mathrm{M}^{+1}, 69\right), 272\left(\mathrm{M}^{+}, 97\right)$, 229 (96), 183 (96), 157 (97), 155 (100), 128 (97), 101 (57), 43 (98).

$\mathrm{N}$-(2-Phenyl-quinolin-3-ylmethyl)-acetamide (11a). A solution of phenyl-[1-phenyl-meth-(E)ylidene]-amine $7 \mathbf{a}(21 \mathrm{mg}, 0.12 \mathrm{mmol})$, aniline $(11.5 \mathrm{mg}, 0.12 \mathrm{mmol}), \mathrm{N}$-acetyl-2-azetine 6 $(11.7 \mathrm{mg}, 0.12 \mathrm{mmol})$ and yttrium triflate $(3 \mathrm{mg}, 5.0 \mathrm{~mol} \%)$ in acetonitrile $(5 \mathrm{~mL})$ was stirred at room temperature overnight. The solution was then boiled under reflux for 7 hours. Workup according to the general procedure gave the titled compound $(27 \mathrm{mg}, 82 \%)$ as a colorourless solid mp 195-198 ${ }^{\circ} \mathrm{C}$, ( $\mathrm{R}_{\mathrm{f}}$ : 0.26; ethyl ethanoate:hexane 7:3,); HRMS (EI): $\mathrm{C}_{18} \mathrm{H}_{16} \mathrm{~N}_{2} \mathrm{O}$ requires $\mathrm{M}^{+}$276.1263, found $\mathrm{M}^{+} 276.1256 ; \mathrm{v}_{\max }(\mathrm{KBr}) / \mathrm{cm}^{-1} 3274,1647,1557,1261,772,706 ;{ }^{1} \mathrm{H} \mathrm{NMR}$ $\left(500.1 \mathrm{MHz}, \mathrm{CDCl}_{3}\right) \delta(\mathrm{ppm}) 1.92(3 \mathrm{H}, \mathrm{s}), 4.52(2 \mathrm{H}, \mathrm{d}, \mathrm{J}=5.9 \mathrm{~Hz}), 5.92(1 \mathrm{H}, \mathrm{br}), 7.44-7.53(6 \mathrm{H}$, 
overlapping m), 7.69(1H, t, J = 8.3 Hz), $7.80(1 \mathrm{H}, \mathrm{d}, \mathrm{J}=8.3 \mathrm{~Hz}), 8.10(1 \mathrm{H}, \mathrm{d}, \mathrm{J}=8.3 \mathrm{~Hz}), 8.16$ $(1 \mathrm{H}, \mathrm{s}) ;{ }^{13} \mathrm{C}$ NMR $\left(125.8 \mathrm{MHz}, \mathrm{CDCl}_{3}\right) \delta(\mathrm{ppm}) 23.1,41.3,126.8,127.3,127.4,126.6(3$ overlapping peaks) (5C), 129.2, 129.71, 129.73, 136.1, 139.9, 147.0, 159.4, 170.0; MS (EI), $\mathrm{m} / \mathrm{z}$ (\%), $276\left(\mathrm{M}^{+}, 32\right), 233$ (21), 217 (87), 206 (19), 91 (32), 84 (100), 43 (50).

$N$-(5-Bromo-2-phenyl-quinolin-3-ylmethyl)-acetamide $11 \mathrm{~h}$ and $N$-(7-Bromo-2-phenylquinolin-3-ylmethyl)-acetamide (11g). A solution of (3-bromo-phenyl)-[1-phenyl-meth- $(E)$ ylidene]-amine (65 mg, $0.25 \mathrm{mmol}), \mathrm{N}$-acetyl-2-azetine 6 (22 mg, $0.23 \mathrm{mmol})$, 3-bromoaniline (46 mg, $0.27 \mathrm{mmol})$ and yttrium triflate $(7 \mathrm{mg}, 5.0 \mathrm{~mol} \%)$ in anhydrous acetonitrile $(10 \mathrm{~mL})$ was stirred overnight at room temperature and then boiled under reflux for 12 hours. Workup according to general procedure gave $N$-(5-bromo-2-phenyl-quinolin-3-ylmethyl)-acetamide 11h (38 mg, 47\%) Rf: 0.55 ; ethyl ethanoate:hexane 1:1,) as a colorourless solid mp $213-214^{\circ}$ and $\mathrm{N}$ (7-bromo-2-phenyl-quinolin-3-ylmethyl)-acetamide $\quad 11 \mathrm{i} \quad(24 \mathrm{mg}, \quad 30 \%) \quad\left(\mathrm{R}_{\mathrm{f}}\right.$ : 0.31 ; ethyl ethanoate:hexane 1:1) as a colorourless solid mp 190-192 ${ }^{\circ} \mathrm{C}$.

Data for 11h. HRMS (EI): $\mathrm{C}_{18} \mathrm{H}_{15} \mathrm{BrN}_{2} \mathrm{O}$ requires $\mathrm{M}^{+}$354.0368, found $\mathrm{M}^{+}$354.0358; $\mathrm{v}_{\max }$ $(\mathrm{KBr}) / \mathrm{cm}^{-1} 3267,1638,1543,1280,774,700 ;{ }^{1} \mathrm{H}$ NMR $\left(500.1 \mathrm{MHz}, \mathrm{CDCl}_{3}\right) \delta(\mathrm{ppm}) 1.96(3 \mathrm{H}$, s), $4.60(2 \mathrm{H}, \mathrm{d}, \mathrm{J}=5.9 \mathrm{~Hz}), 5.87(1 \mathrm{H}, \mathrm{br}), 7.46-7.55(6 \mathrm{H}$, overlapping $\mathrm{m}), 7.80(1 \mathrm{H}, \mathrm{d}, \mathrm{J}=7.5$ $\mathrm{Hz}), 8.07(1 \mathrm{H}, \mathrm{d}, \mathrm{J}=8.5 \mathrm{~Hz}), 8.50(1 \mathrm{H}, \mathrm{s}) ;{ }^{13} \mathrm{C} \mathrm{NMR}\left(125.8 \mathrm{MHz}, \mathrm{CDCl}_{3}\right) \delta(\mathrm{ppm}) 23.1,41.4$, $121.5,126.7,128.6(2 \mathrm{C}), 128.7(2 \mathrm{C}), 128.9,129.2,129.9,130.4,131.1,135.3,139.3,147.7$, 160.1, 169.9; MS (EI), $m / z(\%), 356\left({ }^{81} \mathrm{Br} \mathrm{M}^{+} 50\right), 354\left({ }^{79} \mathrm{Br} \mathrm{M}^{+} 49\right), 311$ (42), 297 (100), 295 (98), 286 (10), 284 (11), 216 (91), 43 (63).

Data for 11i. HRMS (EI): $\mathrm{C}_{18} \mathrm{H}_{15} \mathrm{BrN}_{2} \mathrm{O}$ requires $\mathrm{M}^{+}$354.0368, found $\mathrm{M}^{+} 354.0363 ; \mathrm{v}_{\max }$ $(\mathrm{KBr}) / \mathrm{cm}^{-1} 3279,1646,1259,763,703 ;{ }^{1} \mathrm{H}$ NMR $\left(500.1 \mathrm{MHz}, \mathrm{CDCl}_{3}\right) \delta(\mathrm{ppm}) 1.96(3 \mathrm{H}, \mathrm{s})$, $4.55(2 \mathrm{H}, \mathrm{d}, \mathrm{J}=6.0 \mathrm{~Hz}), 5.71(1 \mathrm{H}, \mathrm{br}), 7.47-7.53(5 \mathrm{H}$, overlapping $\mathrm{m}), 7.63(1 \mathrm{H}, \mathrm{dd}, \mathrm{J}=8.7,1.8$ $\mathrm{Hz}), 7.69(1 \mathrm{H}, \mathrm{d}, \mathrm{J}=8.7 \mathrm{~Hz}), 8.16(1 \mathrm{H}, \mathrm{s}), 8.31(1 \mathrm{H}, \mathrm{d}, \mathrm{J}=1.8 \mathrm{~Hz}) ;{ }^{13} \mathrm{C}$ NMR $(125.8 \mathrm{MHz}$, $\left.\mathrm{CDCl}_{3}\right) \delta(\mathrm{ppm}) 23.2,41.3,123.8,125.9,128.6(2 \mathrm{C}), 128.7,128.74(2 \mathrm{C}), 128.9,130.2,130.3$, 131.7, 135.9, 139.7, 147.6, 160.3, 170.0; MS (EI), $m / z(\%), 356\left({ }^{81} \mathrm{Br} \mathrm{M}^{+} 46\right), 354\left({ }^{79} \mathrm{Br} \mathrm{M}^{+} 47\right)$, 297 (95), 295 (93), 286 (61), 284 (28), 216 (100), 43 (42).

$N$-[2-(4-Hydroxy-phenyl)-quinolin-3-ylmethyl]acetamide (11j). A solution of 4-[(E)-ptolylimino-methyl]-phenol (131 mg, $0.66 \mathrm{mg}$ ), aniline $(62 \mathrm{mg}, 0.67 \mathrm{mmol}), N$-acetyl-2-azetine 6 $(61 \mathrm{mg}, 0.63 \mathrm{mmol})$ and yttrium triflate $(14 \mathrm{mg}, 4.0 \mathrm{~mol} \%)$ in anhydrous acetonitrile $(12 \mathrm{~mL})$ was stirred at room temperature for 24 hours and heated under reflux for 5 hours. Work up according to general procedure gave the titled compound (114 mg, 62\%), as a colorourless solid mp 114-116 ${ }^{\circ} \mathrm{C}$ ( $\mathrm{R}_{\mathrm{f}}$ : 0.22; ethyl ethanoate:hexane:methanol 78:20:2); HRMS (EI): $\mathrm{C}_{18} \mathrm{H}_{16} \mathrm{~N}_{2} \mathrm{O}_{2}$ requires $\mathrm{M}^{+}$292.1212, found $\mathrm{M}^{+}$292.1214; $\mathrm{v}_{\max }(\mathrm{KBr}) / \mathrm{cm}^{-1} 3287,1652,1170,758,845 ;{ }^{1} \mathrm{H}$ NMR $\left(300.1 \mathrm{MHz}, \mathrm{CD}_{3} \mathrm{OD}\right) \delta(\mathrm{ppm}) 1.88(3 \mathrm{H}, \mathrm{s}), 3.27(1 \mathrm{H}, \mathrm{s}), 4.37(2 \mathrm{H}, \mathrm{s}), 6.83(2 \mathrm{H}, \mathrm{d}, \mathrm{J}=8.6$ $\mathrm{Hz}), 7.30(2 \mathrm{H}, \mathrm{d}, \mathrm{J}=8.6 \mathrm{~Hz}), 7.48(1 \mathrm{H}, \mathrm{t}, \mathrm{J}=8.4 \mathrm{~Hz}), 7.60(1 \mathrm{H}, \mathrm{t}, \mathrm{J}=8.4 \mathrm{~Hz}), 7.82(1 \mathrm{H}, \mathrm{d}, \mathrm{J}=$ 8.4), $7.89(1 \mathrm{H}, \mathrm{d}, \mathrm{J}=8.4 \mathrm{~Hz}), 8.20(1 \mathrm{H}, \mathrm{s}) ;{ }^{13} \mathrm{C} \mathrm{NMR}\left(125.8 \mathrm{MHz}, \mathrm{CD}_{3} \mathrm{OD}\right) \delta(\mathrm{ppm}) 21.0,40.7$, 114.9(2C), 126.5, 126.7, 127.2, 127.3, 129.5, 129.9(3C), 130.4, 136.5, 145.3, 158.2, 159.3, 171.9; MS (EI), m/z (\%), 292 (M+ 0.5), 290 (8), 263 (12), 247 (15), 219 (7), 184 (17), 156 (7), 93 (14), 84 (54), 59 (100). 


\section{Acknowledgements}

We would like to thank Queens University for the award of a studentship to Daire Osborne

\section{References and Footnotes}

1. Povarov, L. S. Russ. Chem. Rev. 1967, 36, 656.

2. Buonora, P.; Olsen, J. C.; Oh, T. Tetrahedron 2001, 57, 6099.

3. Behforouz, M.; Ahmadian, M. Tetrahedron 2000, 56, 5259.

4. Povarov, S.; Grigos, V. I.; Makhailov, B. M. Izv. Akad. Nauk. SSSR. 1963, 2039.

5. Povarov, S.; Makhailov, B. M. Izv. Akad. Nauk. SSSR. 1963, 955.

6. Yadav, J. S.; Reddy, B. V. S.; Rao, R. S.; Kumar, S. K.; Kunwar, A. C. Tetrahedron 2002, $58,7891$.

7. Semwal, A.; Nayak, S. K. Syn. Comm. 2006, 36, 227.

8. Laurent-Robert, H.; Garrigues, B.; Dubac, J. Synlett 2000, 1160.

9. Kobayashi, S.; Ishitani, H.; Nagayama, S. Synthesis 1995, 1195.

10. Nagarajan, R.; Perumal, P. T. Syn. Comm. 2001, 31, 1733.

11. Nagarajan, R.; Magesh, C. J.; Perumal, P. T. Synthesis 2004, 69.

12. Nagaiah, K.; Sreenu, D.; Rao, R. S.; Vashishta, G.; Yadav, J. S. Tetrahedron Lett. 2006, 47, 4409.

13. Yadav, J. S.; Reddy, B. V. S.; Reddy, J. S. S.; Rao, R. S. Tetrahedron 2003, 59, 1599.

14. Di Salvo, A.; Spanedda, M. V.; Ourevitch, M.; Crousse, B.; Bonnet-Delpon, D. Synthesis 2003, 2231.

15. Beifuss, U.; Ledderhose, S.; Ondrus, V. Arkivoc 2005, 147.Part 5

16. Hermitage, S.; Howard, J. A. K.; Jay, D.; Pritchard, R. G.; Probert, M. R.; Whiting, A. Org. Biomol. Chem. 2004, 2, 2451.

17. Hermitage, S.; Jay, D. A.; Whiting, A. Tetrahedron Lett. 2002, 43, 9633.

18. Witherup, K. M.; Ransom, R. W.; Graham, A. C.; Bernard, A. M.; Salvatore, M. J.; Lumma, W. C.; Anderson, P. S.; Pitzenberger, S. M.; Varga, S. L. J. Am. Chem. Soc. 1995, 117 , 6682 .

19. Hadden, M.; Nieuwenhuyzen, M.; Osborne, D.; Stevenson, P. J.; Thompson, N. Tetrahedron Lett. 2001, 42, 6417.

20. Hadden, M.; Nieuwenhuyzen, M.; Osborne, D.; Stevenson, P. J.; Thompson, N.; Walker, A. D. Tetrahedron 2006, 62, 3977.

21. Hadden, M.; Nieuwenhuyzen, M.; Potts, D.; Stevenson, P. J.; Thompson, N. Tetrahedron 2001, 57, 5615.

22. Xia, C. F.; Heng, L. S.; Ma, D. W. Tetrahedron Lett. 2002, 43, 9405.

23. Powell, D. A.; Batey, R. A. Org. Lett. 2002, 4, 2913.

24. Batey, R. A.; Powell, D. A. Chem. Comm. 2001, 2362. 
25. Crousse, B.; Begue, J. P.; Bonnet-Delpon, D. J. Org. Chem. 2000, 65, 5009.

26. Savitha, G.; Perumal, P. T. Tetrahedron Lett. 2006, 47, 3589.

27. Jung, M. E.; Choi, Y. M. J. Org. Chem. 1991, 56, 6729.

28. Dave, P. R.; Duddu, R.; Li, J. C.; Surapaneni, R.; Gilardi, R. Tetrahedron Lett. 1998, 39, 5481.

29. Dave, P. R.; Duddu, R.; Surapaneni, R.; Gilardi, R. Tetrahedron Lett. 1999, 40, 443.

30. Stevenson, P. J.; Nieuwenhuyzen, M.; Osborne, D. Chem. Comm. 2002, 444.

31. Osborne, D.; Stevenson, P. J. Tetrahedron Lett. 2002, 43, 5469.

32. The crystal structure was previously deposited with Cambridge Structural Database CSD, CCDC number 174518.

33. Hoffmann, R. W. Chem. Rev. 1989, 89, 1841.

34. Katritzky, A. R.; Strah, S.; Tymoshenko, D. O. J. Heterocycl. Chem. 1999, 36, 755.

35. Stevenson, P. J.; Graham, I. Arkivoc 2003, 139.

36. McKittrick, B. A.; Ma, K.; Huie, K.; Yumibe, N.; Davis, H.; Clader, J. W.; Czarniecki, M.; McPhail, A. T. J. Med. Chem. 1998, 41, 752.

37. Nongkunsarn, P.; Ramsden, C. A. Tetrahedron 1997, 53, 3805.

38. Couture, A.; Deniau, E.; Grandclaudon, P. Synthesis 1994, 953.

39. Hantzsch, A. Ber. Dtsc. Chem. Ges. 1901, 34, 822.

40. Dave, P. R.; Kumar, K. A.; Duddu, R.; Axenrod, T.; Dai, R.; Das, K. K.; Guan, X. P.; Sun, J. G.; Trivedi, N. J.; Gilardi, R. D. J. Org. Chem. 2000, 65, 1207. 